\title{
Electron burst driven by near electric field effects of lower-hybrid launchers
}

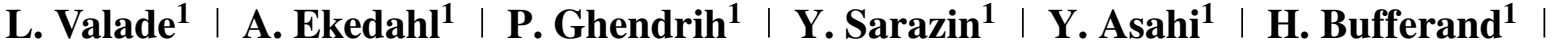

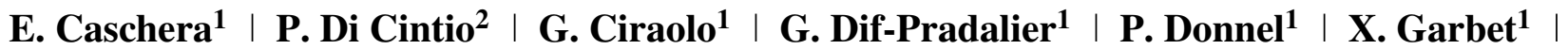

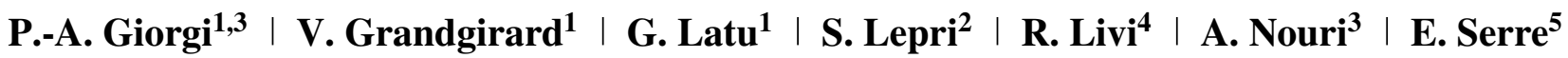

${ }^{1}$ CEA, IRFM, Saint-Paul-lez-Durance, France

${ }^{2}$ Istituto dei Sistemi Complessi, Consiglio

Nazionale delle Ricerche, Sesto Fiorentino, Italy

${ }^{3}$ Aix Marseille Univ, CNRS, Centrale Marseille,

I2M, Marseille, France

${ }^{4}$ Dipartimento di Fisica e Astronomia, Università

di Firenze, Sesto Fiorentino, Italy

${ }^{5}$ Aix Marseille Univ, CNRS, Centrale Marseille,

M2P2, Marseille, France

*Correspondence

P. Ghendrih, IRFM-513, CEA-Cadarache, F-13108

Saint-Paul-lez-Durance, France.

Email: philippe.ghendrih@cea.fr

Funding Information

This research was supported by the A*MIDEX.

EUROfusion, ER-WP15/IPP-01.
Hotspot generation by lower-hybrid (LH) launchers is found to be governed by a resonance in the plasma electric field response to the external drive. The kinetic analysis in $1 \mathrm{D}-1 \mathrm{~V}$ in the parallel direction allows one to compute the amplification effect for small amplitude of the external drive. The resonant Lorentzian response distorts the distribution function. An island structure is formed in the suprathermal part at the phase velocity of the external electrostatic drive. The non-linear features enhance the plasma response, driving overlap effects between multiple waves at rather low amplitude. The onset of a plateau in the distribution function, with extent reaching one thermal velocity, is already obtained when the standard overlap condition is achieved. The sensitivity of the resonance to plasma parameters and large variation of the amplification magnitude can compensate the fast radial decay of the small-scale features generated by the LH launchers, which are responsible for the interaction with the cold electrons. This mechanism can trigger hotspot generation further in the scrape-off layer than otherwise expected.

\section{KEYWORDS}

hot electron plateau, hotspot generation, island overlap, kinetic, lower hybrid, resonance, scrape-off layer

\section{1 | HOTSPOTS DURING LOWER-HYBRID OPERATION}

Lower-hybrid (LH) launchers installed in the scrape-off layer (SOL) plasma generate near electric fields that can accelerate SOL electrons. ${ }^{[1-3]}$ This mechanism is understood as the drive for hotspot generation on field lines connected to the launchers. ${ }^{[4]}$ Experimental evidence supports the latter fact, and theoretical analysis provides a qualitative understanding. ${ }^{[5,6]}$ Recent experimental analysis has underlined the importance of turbulence in the hotspot generation mechanism. ${ }^{[7]}$ Open questions related to this problem are the optimum between density and distance in accelerating SOL electrons on the one hand and hotspot properties on the other hand. ${ }^{[8,9]}$ Regarding the latter, transport along the field line will determine the splitting between the ion and electron channel and, consequently, the coupling to the sheath prior to deposition on a plasma-facing component. The LH launchers generate waves in the Gigahertz range, typically from 2.5 to $8 \mathrm{GHz}$ in present experiments, the frequency increasing with the magnitude of the toroidal field of the device. In devices with strongly constrained access to the plasma, either high field devices such as Alcator C-Mod ${ }^{[10]}$ or medium-sized devices with superconducting coils as WEST Tungsten (W) Environment 
in Steady-state Tokamak, ${ }^{[11,12]}$ heating and current drive must rely on waves as the conventional neutral beam heating is made difficult. In that framework, the so-called LH system has several assets, especially to sustain plasma current in long pulse operation. However, the occurrence of hotspots in regions connected to the launchers in the parallel direction can have severe impact on the integrity of plasma-facing components, as documented quite early on ASDEX ${ }^{[1]}$ and since in several other devices. ${ }^{[2,4,7]}$ While the LH frequency is well defined, the wave vector is determined by the mechanical structure of the LH wave guides as well as the radial propagation properties into the plasma. The sharp jump between the electric field in the launchers material, limited to a skin depth, and that in the wave guide significantly broadens the spectrum compared to the useful wave range for interaction with hot core electrons. ${ }^{[8]}$

The paper addresses the kinetic response of the electrons characterized by a resonance, Section 2 . The impact on the generation of a plateau in the distribution function is described in Section 3. Implication and future work is addressed in the Conclusion and Discussion Section 4.

\section{2 | RESONANT RESPONSE OF KINETIC ELECTRONS TO HIGH-FREQUENCY ELECTRIC FIELDS}

The resonance between electrons and waves at the LH frequency, launched at $3.7 \mathrm{GHz}$ in WEST, occurs for velocities $v / V_{\text {the }}=$ $4.84 \cdot 10^{-1} /\left(k \lambda_{D} \sqrt{n / 10^{18} \mathrm{~m}^{-3}}\right)$, where $V_{\text {the }}$ is the electron thermal velocity, and $k \lambda_{D}$ the wave length of the wave normalized by the Debye length $\lambda_{D}$. At low-density $n=10^{18} \mathrm{~m}^{-3}$ and long wave length $k \lambda_{D}=0.1$, the resonant electrons with the LH wave are therefore suprathermal $v / V_{\text {the }}=4$.8. In such a regime, electrons have small collisionality and must therefore be described kinetically. The appropriate description of the plasma is therefore kinetic, completed by Maxwell equations to determine the electromagnetic field. Furthermore, for wave lengths comparable to the Debye scale, one cannot assume the quasineutral limit. To simplify the problem, we consider a single direction in position and velocity corresponding to the parallel motion of the electrons and restrict the problem to the electrostatic limit, taking into account an external drive due to the LH launcher. In the simplest form, we thus address a 1D-1V kinetic model with the standard Vlasov-Poisson system. The Eulerian version, pseudo-spectral in both velocity and position directions, of the VOICE code is used in the present work. Some aspect of collisions can be taken into account by a Bhatnagar, Gross and Krook (BGK) restoring force towards the initial distribution function. With standard normalization, plasma frequency for time, Debye length for length scales, reference density for distribution functions and thermal velocities for particle velocity, the two species set of equations is then:

$$
\begin{gathered}
\partial_{t} f_{e}+v \partial_{x} f_{e}+\partial_{x}\left(\phi+\phi_{e x t}\right) \partial_{v} f_{e}=-\sigma_{e}\left(f_{e}-f_{e, 0}\right), \\
\partial_{t} f_{i}+\sqrt{\frac{m_{e}}{m_{i}}}\left(v \partial_{x} f_{i}-\partial_{x}\left(\phi+\phi_{e x t}\right) \partial_{v} f_{i}\right)=-\sigma_{i}\left(f_{i}-f_{i, 0}\right), \\
\partial_{x}^{2} \phi(x, t)=\int d v^{\prime} f_{e}\left(x, v^{\prime}, t\right)-\int d v^{\prime \prime} f_{i}\left(x, v^{\prime \prime}, t\right) .
\end{gathered}
$$

Here, $-\partial_{x} \phi_{\text {ext }}$ is the driving external electric field and $\phi$ the self-consistent electric potential induced in the plasma. Note that the velocity normalization is different for each species so that the mass effect is not taken into account in the distribution functions but appears as the square root of the mass ratio in the evolution equation of the ion species, consistent with a much slower evolution rate for ions than for electrons. As a consequence, when addressing short time-scale effects for the electrons, one can readily work in the frozen ion limit. In the literature, such an externally driven Vlasov equation is also addressed as the KEEN wave ${ }^{[13,14]}$ (Kinetic Electron Electrostatic Nonlinear wave). However, the emphasis is then put on a second phase of self-sustained self-organization once the external drive is set back to zero.

The linear response for small amplitude of $\phi_{\text {ext }}$ has been analysed. ${ }^{[8]}$ It is characterized by a resonant effect that can be seen in the amplitude of the electric potential generated by the plasma.

$$
k^{2} \hat{\phi}(\omega, k)=-\left(G_{e}\left(\omega / k, \sigma_{e} / k, f_{e, 0}\right)+G_{i}\left(\omega / k_{i}, \sigma_{i} / k_{i}, f_{i, 0}\right)\right)\left(\hat{\phi}(\omega k)+\widehat{\phi}_{\text {ext }}(\omega, k)\right) .
$$

This response is computed in Fourier space, $\hat{\phi}(\omega, k)$ and $\hat{\phi}_{\text {ext }}(\omega, k)$ being the Fourier transform of the external and self-consistent electric potentials, respectively. The response function $G_{a}\left(\omega / k_{a}, \sigma_{a} / k_{a}, f_{a}\right)$ for species $a$ is defined by:

$$
G_{a}\left(\omega / k_{a}, \sigma / k_{a}, f_{a}\right)=\int d v \frac{1}{v-\omega / k_{a}-i \sigma / k_{a}}\left(-\partial_{v} f_{a}(v)\right),
$$



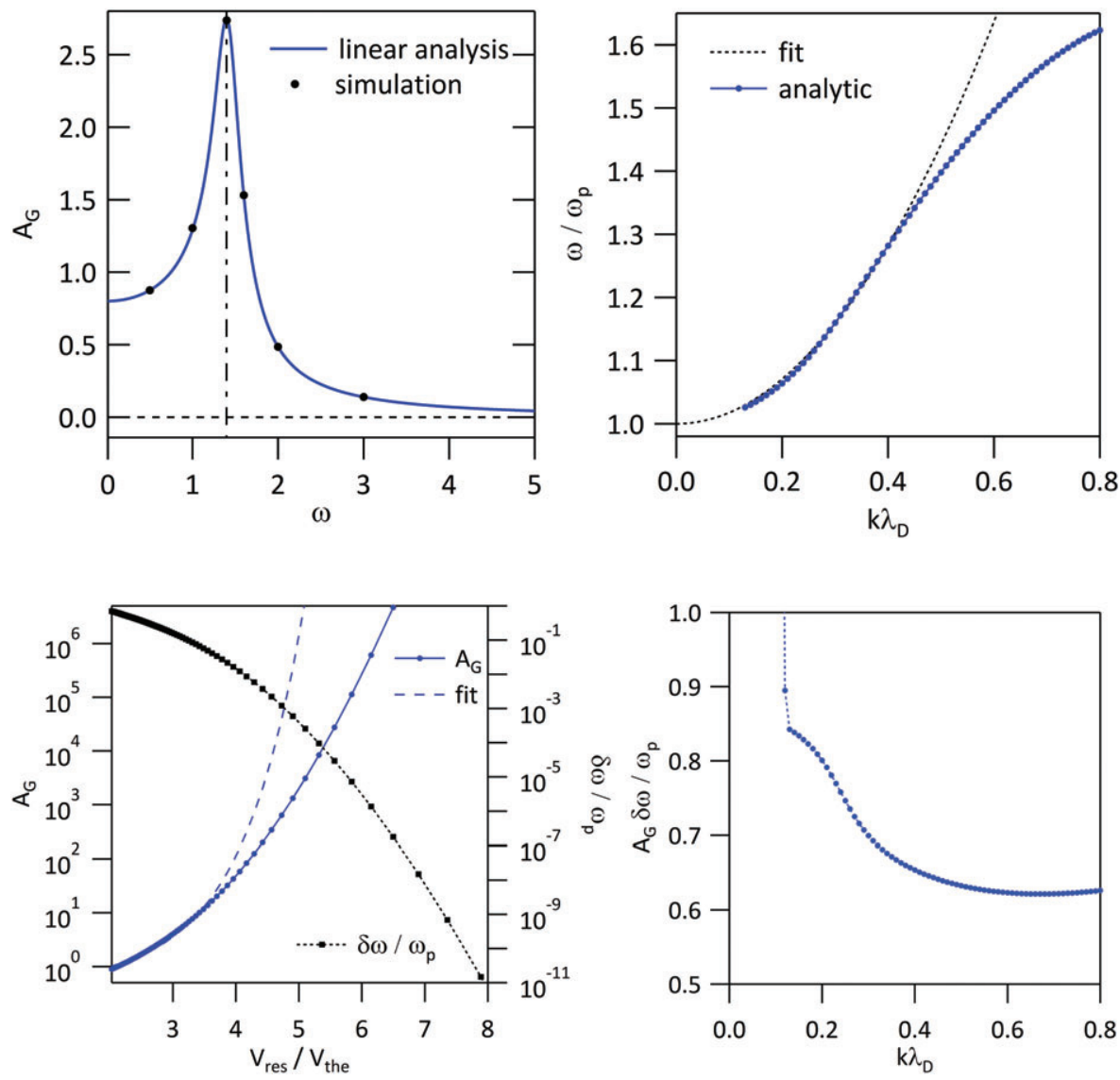

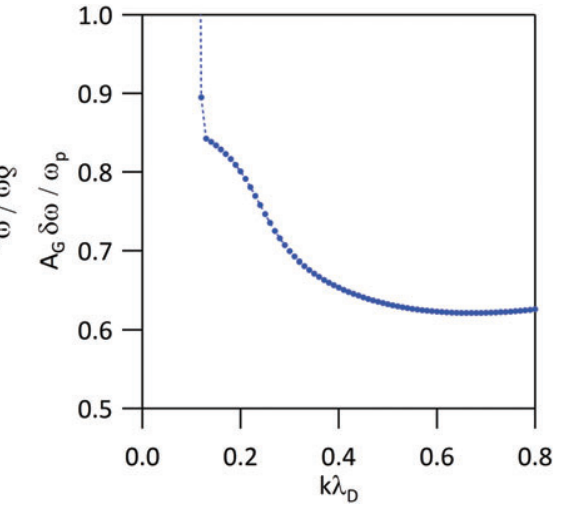

FIGURE 1 Left Hand side: Amplitude ratio $A_{G}$ between the potential generated by the plasma and that of the external drive for $k=0.5$ as a function of $\omega$, neglecting the ion contribution and for $\sigma=0$. The linear approximation yields (blue) curve, and VOICE simulations are indicated by the closed (black) circles. The VOICE simulation is performed with $\left|\widehat{\phi_{\text {ext }}}(\omega, k)\right|=10^{-5}$. Right Hand Side: Dependence of the resonant pulsation $\omega$ on the wave vector $k$
FIGURE 2 LHS: Variation of the amplitude ratio $A_{G}$ between the potential generated by the plasma and that of the external drive and variation of the width of the resonance $\delta \omega$ for increasing phase velocities $V_{\text {res }}=\omega / k$, neglecting the ion contribution and for $\sigma=0$. RHS: Product of the resonance width and gain $A_{G} \delta \omega$

where $k_{a}=k / \sqrt{m_{a} / m_{e}}$. For Maxwellian distribution functions, the function $G_{a}$ is related to the plasma function. Because of the mass ratio effect, which appears via $k_{a}$, the ion contribution in 2a is quite small and can be neglected. The amplitude ratio $A_{G}=|\hat{\phi}(\omega, k)| /\left|\hat{\phi}_{\text {ext }}(\omega, k)\right|$ is shown on the left-hand side (LHS) of Figure 1.

One recovers expected features; at high-frequency $\omega \gg 1$, the electron do not respond to the external field, while in the adiabatic regime $\omega \ll 1$, there is an order 1 response. Near the plasma frequency, one finds the resonant feature, with a Lorentzian-like shape. As expected for a Lorentzian, the resonance is also characterized by a change of phase by $\pi$ between the low-frequency regime, where the plasma electrons tend to screen the external electric potential, and the high-frequency regime, where the electric potential generated by the plasma tends to be in phase with the external drive. The VOICE simulation data (black closed circles in Figure 1 LHS) is in very good agreement with the analytical formula. Using the latter, one can then investigate the dependence of the resonant pulsation $\omega$ on the wave vector $k$, Figure 1 right-hand side (RHS). One finds an asymptotic quadratic dependence in $k$ of $\omega-1$ at small $k$ as expected for Langmuir waves. This regime holds when increasing the wave vector up to the normalized value of $k \approx 0.4$.

Given the resonance value of $\omega$ and $k$, one readily defines the velocity of the electrons in phase with the wave $V_{\text {res }}=\omega / k$, where $V_{\text {res }}$ is normalized by the electron thermal velocity. Given the rather small range of variations of $\omega$, the resonant velocity behaves typically as $1 / k$. Using the phase velocity as a key parameter, one can then analyse the change in peak half width $\delta \omega$ and gain magnitude $A_{G}$ as a function of $V_{\text {res }}$, Figure 2 LHS. One finds that the gain increases faster than exponential but not quite as fast as an exponential of exponential (fit indicated by the dashed curve, adjusted to match the behaviour at low $V_{\text {res }}$ ). Conversely, the width of the resonance decreases faster than exponentially. These very rapid variations lead to extreme values of the gain and width, the latter corresponding to the limit of maximum computer resolution. This limitation is observed in Figure 2 RHS, where the product gain by half width is plotted versus the wave vector $k$. This product is nearly constant but for the points at lowest values of $k$, which depart from the smooth variation as the limit of $\delta \omega$ in numerical resolution is reached. The points with too narrow resonance features are removed from the subsequent plots.

\section{3 | RESPONSE TO TWO WAVES}

Unlike the case of LH, where the generators determine a given frequency and where the finite size effects of the launcher enlarges the wave vector spectrum, here, we shall analyse the response to waves with the same wave vector but different pulsations. 
FIGURE 3 LHS: Trace of the electric potential generated by the plasma at position $x_{\text {mid }}$ corresponding to the centre of the box. RHS: Variation with velocity of the time-averaged distribution function at the same location
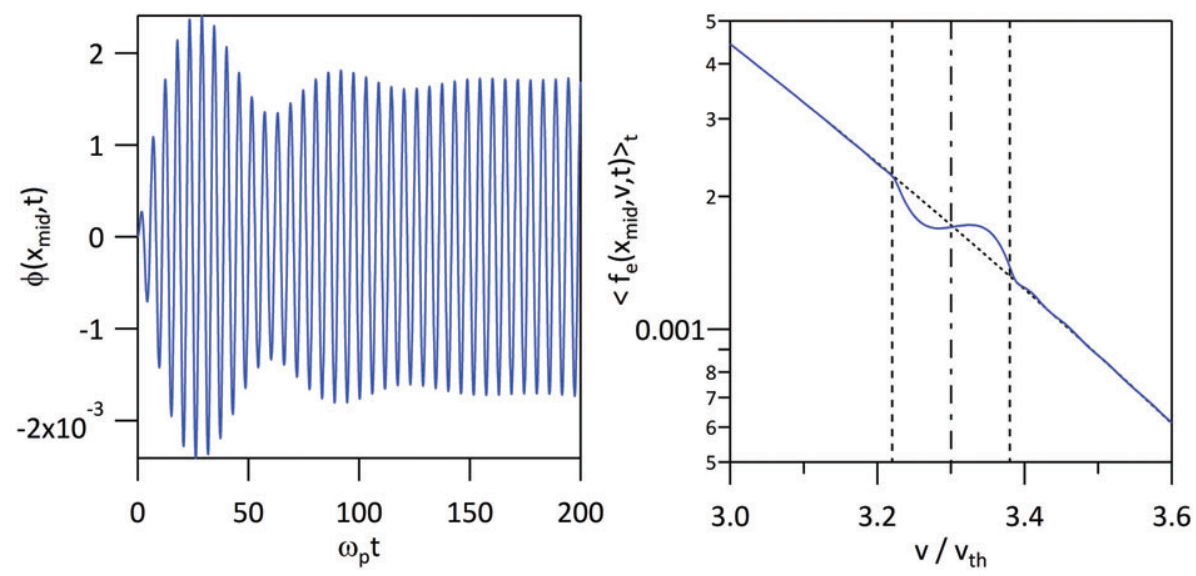

This approach is also quite suitable for the direct use of the chaos onset criteria known as the Chirikov parameter, ${ }^{[15]}$ which is the standard approach when estimating the onset of large-scale chaos. The various modes are then characterized by their phase velocity, in practice determined by different pulsations and an island width, proportional to the square root of the amplitude of the electric potential, with no change in space periodicity. The argument inspired by the transition to chaos is to assume that when two neighbouring modes interact non-linearly (the condition being known as the overlap criterion), a plateau is created in the distribution function that corresponds to the depletion of the slow particle region and population of the fast particle region of the phase space. To handle this situation, the values considered are that of the external field, hence for two phase velocities of the interacting modes and the associated electric potentials that define the island widths. The very strong response of the electric potential generated by the collective behaviour of the plasma modifies this picture quite significantly as the width must now be computed taking into account the plasma response, for which the gain can be so important that it dwarfs the width effect induced by the driving electric potential.

In order to recover a situation that is reminiscent of that of overlapping modes in phase space, we consider two modes that are symmetric with respect to the resonance. For this analysis, we set $k=1 / 3$ such that the resonance pulsation is $\omega_{0}=1.2$ and phase velocity is 3.6 and consider two modes with pulsation $\omega_{1}=1.1$ and $\omega_{2}=1.3$, with phase velocity of 3.3 and 3.9, respectively. These, being symmetric with respect to $\omega_{0}$, have nearly equal linear amplification factor, very close to 4.2 . The island width in velocity space is then equal to $2 \sqrt{A_{G} \phi_{\text {ext }}}, \approx 4.1 \sqrt{\phi_{\text {ext }}}$. From the linear analysis, the Chirikov overlap criterion, when the sum of the two island widths is equal to the separation between the islands, is thus expected to be reached for $\phi_{\text {ext }}=0.532510^{-2}$.

To analyse the VOICE simulation output, let us first consider the case $\phi_{\text {ext }}=0.0410^{-2}$ and the single mode $\omega_{1}$. The time trace of the potential response is shown in Figure 3, LHS. The behaviour of the electric potential can be separated into a high-frequency contribution at $\omega_{1}$ and a low-frequency envelop that also exhibits a time dependence, a growth first and then what could be damped oscillations. While the linear calculation allows one to determine the plasma response in terms of the electric potential, it is important to underline that one cannot compute a steady-state solution for the distribution function $f_{e}$. Indeed, the particle-wave interaction drives a diverging filamentation process. This effect is smeared out by taking the time average once the main transient effects are ended, Figure 3 RHS. For the latter, one finds that $\left\langle f_{e}\right\rangle$ hardly departs from the initial Maxwellian (dashed curve) and that the departure from the Maxwellian is localized at the phase velocity of the mode (vertical dash-dot curve at $V_{1}=3.3$ ) and extends within the range determined by the island width (vertical dashed lines). The response for the distribution function takes the form of a localized flattening at the resonance. When no averaging is performed, one readily notices that the distribution function exhibits very fine structures localized in this region of phase space as well as a global oscillation at the driving frequency.

Comparing the cases with a drive at pulsation $\omega_{1}=1.1$ and $\omega_{2}=1.3$ with the same amplitude $\phi_{\text {ext }}=0.0410^{-2}$ indicates that $\delta f_{e}$ is characterized by the same structure and extent of the perturbed region, in agreement with the same amplification of the electric potential due to the plasma response $4 \phi_{\text {ext }}=4 \times 0.0410^{-2}$, in agreement with the linear analysis, shifted in velocity due to the difference in phase velocity but with a ratio in the amplitude of $\left\langle\delta f_{e}\right\rangle_{t}$ of 3.9, while the ratio of the Maxwellian for the two values $V_{1}=3.3$ and $V_{2}=3.9$ is of the order 8.7. Although one expects the same value for $\delta f_{e} / f_{e}$, one finds that $\left\langle\delta f_{e}\right\rangle_{t} /\left\langle f_{e}\right\rangle_{t}$ varies by a factor of order 2 . Increasing the amplitude of the drive to $\phi_{e x t}=0.1610^{-2}$ and considering the simulation of either $\omega_{1}$ or $\omega_{2}$ indicates that the symmetry in the shape of $\left\langle\delta f_{e}\right\rangle_{t}$ is lost (Figure 4). An important feature is that a response develops in the vicinity of $V_{0}=\omega_{0} / k$, beyond the island separatrix estimated on the basis of the linear analysis. This feature is far more pronounced for the drive at $\omega_{2}$ than for $\omega_{1}$. A tentative explanation is the relative location of the phase velocity of the mode, that of the resonance $V_{0}$ and the bulk of the distribution function. Indeed, the Langmuir waves are essentially governed by the bulk of the distribution function (and are consequently recovered in the fluid approximation). These are coupled to the velocity layers neighbouring the phase velocity, which drives the amplification. For velocities larger than the phase velocity, the 

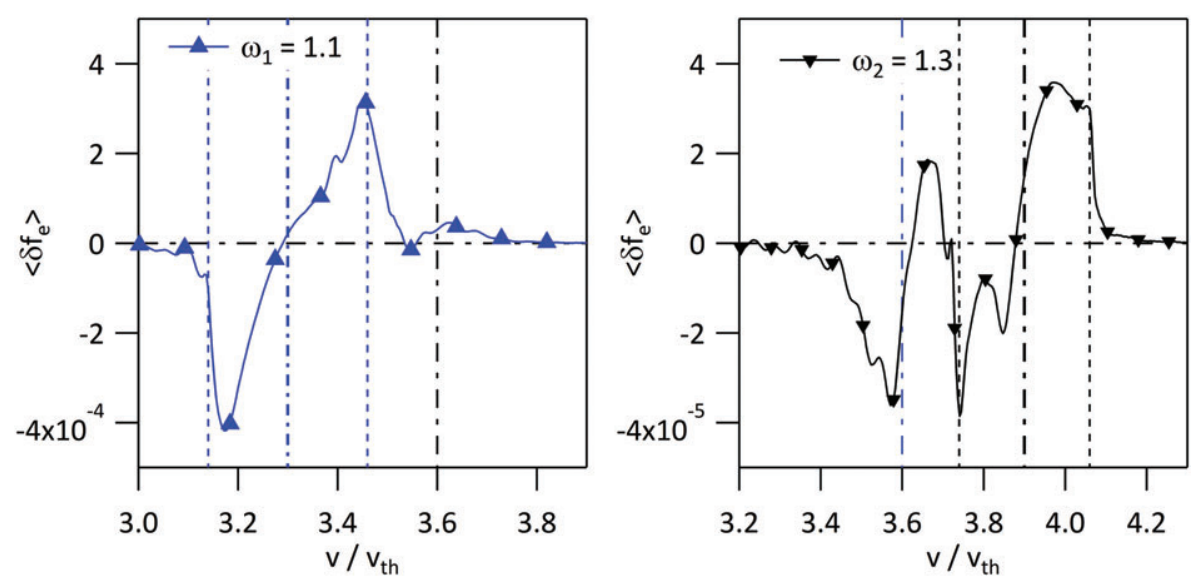

FIGURE 4 Time average of the response of the distribution function compared to the initial Maxwellian $\left\langle\delta f_{e}\right\rangle_{t}$ for an external drive with amplitude $0.1610^{-2}$ for two different simulations. Bold dash-dot vertical lines correspond to phase velocity of the modes, the thin dash-dot vertical lines indicate the phase velocity at pulsation $\omega_{0}$ and the dashed vertical lines the extent as expected from the linear analysis. LHS: For pulsation, $\omega_{1}=1.1$. RHS: For pulsation, $\omega_{2}=1.3$
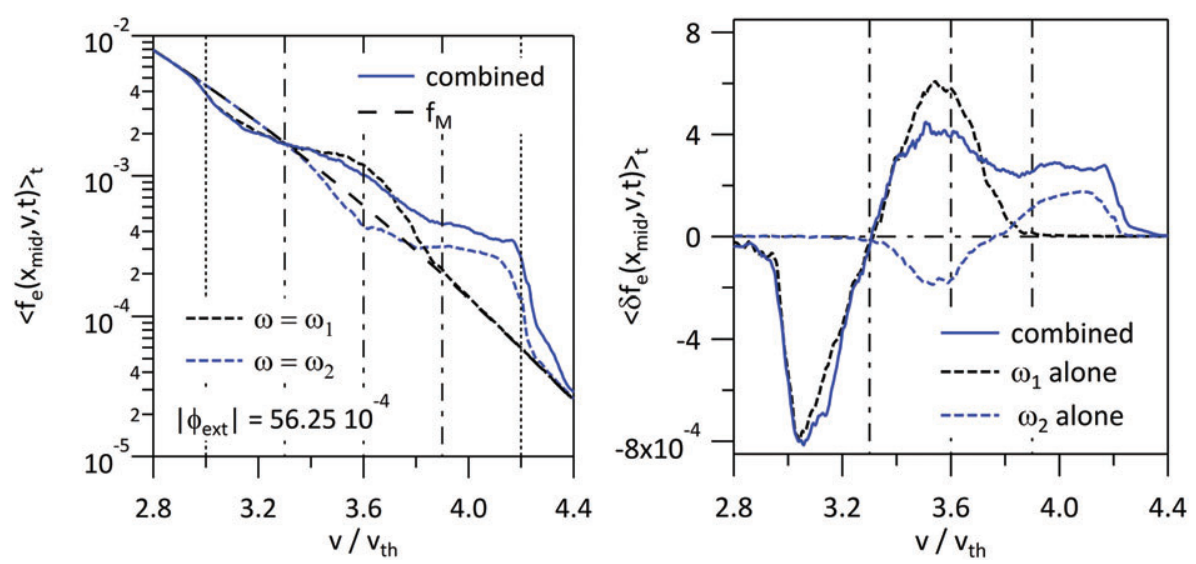

FIGURE 5 LHS: Time average of the response of the distribution function and initial Maxwellian for an external drive with amplitude $56.2510^{-4}$ for three different simulations bold large dash (black) initial Maxwellian, thin short dash (blue) single frequency at $\omega_{2}$, boldshort dash (black) single frequency at $\omega_{1}$, plain (blue) curve with the two frequencies $\omega_{1}$ and $\omega_{2}$. Bold dash-dot vertical lines correspond to phase velocity of the modes, the thin dash-dot vertical lines indicate the phase velocity at pulsation $\omega_{0}$ and the dashed vertical lines the extent as expected from the linear analysis. RHS same simulation results and trace convention for $\left\langle\delta f_{\mathrm{e}}\right\rangle_{\mathrm{t}}$.

response is small. As a consequence, when the plasma-wave interaction region is ahead of the resonance, the latter is weakly modified. Conversely, when the plasma-wave interaction region lies beyond the resonance, a response at the resonance $V_{0}$ can be generated provided the detuning between the excited mode and the resonance is not too large, typically of order 0.1 in pulsation, but also because the amplification at the resonance is quite large $\left(A_{G} \approx 16\right)$, in the present case four times larger than at the chosen phase velocity $\left(A_{G} \approx 4\right)$.

Increasing the driving mode amplitude to reach the overlap threshold according to the linear analysis leads to the formation of a plateau in the distribution function when the two modes $\omega_{1}$ and $\omega_{2}$ are present, Figure 5 LHS. This is a signature of non-linear effects driving a mixing of the distribution function over a large region of velocity space, here typically from 2.9 to 4.1 $V_{\text {the }}$. The plateau is built by depleting the low velocity region, typically from 2.9 to 3.1 . These particles are accelerated by the electric field to higher velocities due to the combined effect of the two modes, thus generating the plateau in the distribution function. Although the overall distortion of the distribution function is governed by non-linear effects, it appears to be rather well approximated as the superimposed results of the distortion due to the two modes when considered independently, Figure 5 RHS. As already discussed for the case with lower amplitude drive, these cases with a single mode are already characterized by non-linear effects that spread the effect of either perturbation in the whole resonance region.

\section{4 | DISCUSSION AND CONCLUSION}

The interplay of electrons with LH waves in the edge plasma of magnetic confinement devices, addressed in the kinetic framework for the dynamics parallel to the magnetic field, is characterized by a resonance when the amplitude of the external drive is small. The electric field can be amplified by several orders of magnitude when resonance conditions are reached. This collective plasma response modifies the standard picture of island overlap that has been used when describing the generation of a fast electron tail. The non-linearity associated with that response tends to distort the distribution function in the phase space region towards the velocities associated with the largest linear amplification. This non-linear response leads to large-scale velocity transport and the appearance of a plateau in the electron distribution function at lower external perturbation than expected.

In the present analysis, to facilitate the simulations, we have considered a fixed wave vector and tuneable frequency. Regarding LH experiments, one has, in fact, a fixed frequency but a broad and space-dependent spectrum of wave vectors. The scaling 
dependence for the wave vectors are typically $k \lambda_{D} \leq 0.5 / \sqrt{n / 10^{18} \mathrm{~m}^{-3}}$. The small-scale structures of the electric field are then responsible for the interaction with the SOL plasma. These high multi-polar components decay exponentially in the radial direction away from the LH launcher. While such a feature should locate the interaction region of the neighbourhood of the LH launcher, the more-than-exponential sensitivity of the resonance feature can overcome the geometrical screening of the small scales of the driving electric field. Compared to the reference resonance discussed in this paper with $k \lambda_{D}=1 / 3$ and $v_{\phi} / V_{\text {the }}=3.6$, a several orders of magnitude increase in the plasma response can be achieved by increasing the density and slightly lowering the electron temperature. The occurrence of cold plasma blobs in the far SOL, or divertor operation, can therefore create conditions for such highly non-linear behaviour.

Finally, it is to be underlined that these effects correspond to a wave-particle interaction in the suprathermal tail of the electron distribution function. The corresponding density of accelerated electrons is quite small so that the expansion of the hot electrons only requires a small DC-restoring electric field to drive a return current to quasineutrality. As a consequence, this effect should govern only a negligible acceleration of the ions. The LH hotspot generation in this regime would then be governed by collisionless electron heat transport due to the evaporation of the fast electrons. This point is to be further discussed in following papers.

\section{ACKNOWLEDGMENTS}

This work has been carried out thanks to the support of the A*MIDEX project (ANR-11-IDEX-0001-02) funded by the "Investissements d'Avenir" French Government programme, managed by the French National Research Agency (ANR). This work has been carried out within the framework of the EUROfusion Consortium and has received funding from the Euratom research and training programme 2014-2018 under grant agreement No 633053 for the projects ER-WP15/IPP-01, WP17-ENR-CEA-01 and WP17-ENR-CEA-03. The views and opinions expressed herein do not necessarily reflect those of the European Commission.

\section{REFERENCES}

[1] The ASDEX-Team, T. E. Evans, J. Neuhauser, F. Leuterer, E. R. Müller, J. Nucl. Mater. 1990, 176-177(Suppl. C), 202.

[2] M. Goniche, D. Guilhem, P. Bibet, P. Froissard, X. Litaudon, G. Rey, J. Mailloux, Y. Demers, V. Fuchs, P. Jacquet, J. H. Harris, J. T. Hogan, Nucl. Fusion 1998, $38(6), 919$.

[3] J. Hillairet, D. Voyer, A. Ekedahl, M. Goniche, M. Kazda, O. Meneghini, D. Milanesio, M. Preynas, Nucl. Fusion 2010, 50, 125010.

[4] K. M. Rantamaki, V. Petrzilka, P. Andrew, I. Coffey, A. Ekedahl, K. Erents, V. Fuchs, M. Goniche, G. Granucci, E. Jo_rin, S. J. Karttunen, P. Lomas, J. Mailloux, M. Mantsinen, M.-L. Mayoral, D. C. McDonald, J.-M. Noterdaeme, V. Parail, A. A. Tuccillo, F. Zacek, Contributors to the EFDA-JET Workprogramme, Plasma Phys. Controlled Fusion 2005, 47, 1101.

[5] V. Fuchs, M. Goniche, Y. Demers, P. Jacquet, J. Mailloux, Phys. Plasmas 1996, 3, 4023.

[6] V. Fuchs, M. Goniche, Y. Demers, P. Jacquet, J. Mailloux, Phys. Plasmas 1997, 4, 1176.

[7] J. P. Gunn, V. Fuchs, V. Petrzilka, A. Ekedahl, N. Fedorczak, M. Goniche, J. Hillairet, Nucl. Fusion 2016, $56,036004$.

[8] L. Valade, E. Caschera, P. Ghendrih, Y. Sarazin, A. Ekedahl, J. Phys.: Conf. Ser. 2016, 775, 012015.

[9] V. Petrzilka, V. Fuchs, J. Gunn, N. Fedorczak, A. Ekedahl, M. Goniche, J. Hillairet, P. Pavlo, Plasma Phys. Controlled Fusion 2011, 53 , 054016.

[10] J. R. Wilson, R. Parker, M. Bitter, P. T. Bonoli, C. Fiore, R. W. Harvey, K. Hill, A. E. Hubbard, J. W. Hughes, A. Ince-Cushman, C. Kessel, J. S. Ko, O. Meneghini, C. K. Phillips, M. Porkolab, J. Rice, A. E. Schmidt, S. Scott, S. Shiraiwa, E. Valeo, G. Wallace, J. C. Wright, Alcator C-Mod Team, Nucl. Fusion 2009, 49, 115015.

[11] J. Bucalossi, M. Missirlian, P. Moreau, F. Samaille, E. Tsitrone, D. van Houtte, T. Batal, C. Bourdelle, M. Chantant, Y. Corre, X. Courtois, L. Delpech, L. Doceul, D. Douai, H. Dougnac, F. Faïsse, C. Fenzi, F. Ferlay, M. Firdaouss, L. Gargiulo, P. Garin, C. Gil, A. Grosman, D. Guilhem, J. Gunn, C. Hernandez, D. Keller, S. Larroque, F. Leroux, M. Lipa, P. Lotte, A. Martinez, O. Meyer, F. Micolon, P. Mollard, E. Nardon, R. Nouailletas, A. Pilia, M. Richou, S. Salasca, J.-M. Travère, Fusion Eng. Des. 2014, 89(7), 907 Proceedings of the 11th International Symposium on Fusion Nuclear Technology-11 (ISFNT-11) Barcelona, Spain, 15-20 September, 2013.

[12] C. Bourdelle, J. F. Artaud, V. Basiuk, M. Bécoulet, S. Brémond, J. Bucalossi, H. Bufferand, G. Ciraolo, L. Colas, Y. Corre, X. Courtois, J. Decker, L. Delpech, P. Devynck, G. Dif-Pradalier, R. P. Doerner, D. Douai, R. Dumont, A. Ekedahl, N. Fedorczak, C. Fenzi, M. Firdaouss, J. Garcia, P. Ghendrih, C. Gil, G. Giruzzi, M. Goniche, C. Grisolia, A. Grosman, D. Guilhem, R. Guirlet, J. Gunn, P. Hennequin, J. Hillairet, T. Hoang, F. Imbeaux, I. Ivanova-Stanik, E. Jo_rin, A. Kallenbach, J. Linke, T. Loarer, P. Lotte, P. Maget, Y. Marandet, M. L. Mayoral, O. Meyer, M. Missirlian, P. Mollard, P. Monier-Garbet, P. Moreau, E. Nardon, B. Pégourié, Y. Peysson, R. Sabot, F. Saint-Laurent, M. Schneider, J. M. Travère, E. Tsitrone, S. Vartanian, L. Vermare, M. Yoshida, R. Zagorski, JET Contributors, Nucl. Fusion 2015, 55, 063017.

[13] T. W. Johnston, Y. Tyshetskiy, A. Ghizzo, P. Bertrand, Phys. Plasmas 2009, 16, 042105.

[14] B. Afeyan, F. Casas, N. Crouseilles, A. Dodhy, E. Faou, M. Mehrenberger, E. Sonnendrücker, Eur. Phys. J. D 2014, 68(10), 295.

[15] B. V. Chirikov, Phys. Rep. 1979, 52(5), 263. 